\title{
Robotic-assisted vs conventional surgery in medial unicompartmental knee arthroplasty: a clinical and radiological study
}

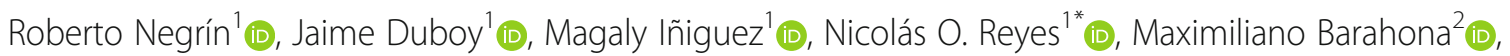
Gonzalo Ferrer ${ }^{1}\left(\mathbb{D}\right.$, Carlos Infante $e^{1,2}$ (D) and Nicolás Jabes ${ }^{1}$ (D)

\begin{abstract}
Background: The use of unicompartmental knee arthroplasty (UKA) has increased and new technologies have been developed to improve patient survival and satisfaction, soft tissue balance, alignment, and component size. Robot-assisted systems offer an increase in surgical precision and accuracy. The purpose of this study is to evaluate the precision of component position using five radiological parameters in conventional and robotic-assisted medial UKA using the NAVIO system.

Methods: A cohort study was designed for patients who underwent medial UKA between April 2017 and March 2019 in a single center. Patients were allocated in the conventional (UKA-C) or robotic-assisted (UKA-R) group. The variables analyzed were age, gender, affected knee side, length of hospital stay, surgical time, and radiological measurements such as anatomical medial distal femoral angle (aMDFA), anatomical medial proximal tibial angle (aMPTA), tibial slope, the sagittal femoral angle, and the component size. A target was defined for each measurement, and a successful UKA was defined if at least four radiological measures were on target after surgery. Also, patients' reported outcomes were evaluated using the Oxford Knee Score (OKS) and a numeric rating scale (NRS) for pain.

Results: Thirty-four patients were included, 18 of them underwent UKA-R. The success rate for UKA in the UKA-R group was 87\%; meanwhile, in the UKA-C group this was 28\%, this difference was significant and powered (Fisher's exact test, $p=0.001 ; 1-\beta=0.95)$. Also, a 5 -point difference in favor of the UKA-R group in the median OKS ( $p=$ $0.01)$, and a significantly lower median NRS for pain $(p<0.000)$ were found after surgery.

Conclusions: UKA-R achieved more precision in the radiological parameters' measure in this study. Also, UKA-R has a trend towards a better OKS and a lower NRS for pain at short-term follow-up.
\end{abstract}

Keywords: Unicompartmental knee, Robotic-assisted surgery

* Correspondence: nicoreyes|@gmail.com

'Department of Orthopedics and Traumatology, Clinica Las Condes, Estoril 450, Las Condes, Santiago, Chile

Full list of author information is available at the end of the article

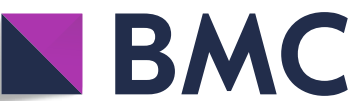

Part of Springer Nature
(9) The Author(s). 2021 Open Access This article is licensed under a Creative Commons Attribution 4.0 International License, which permits use, sharing, adaptation, distribution and reproduction in any medium or format, as long as you give appropriate credit to the original author(s) and the source, provide a link to the Creative Commons licence, and indicate if changes were made. The images or other third party material in this article are included in the article's Creative Commons licence, unless indicated otherwise in a credit line to the material. If material is not included in the article's Creative Commons licence and your intended use is not permitted by statutory regulation or exceeds the permitted use, you will need to obtain permission directly from the copyright holder. To view a copy of this licence, visit http://creativecommons.org/licenses/by/4.0/ The Creative Commons Public Domain Dedication waiver (http://creativecommons.org/publicdomain/zero/1.0/) applies to the data made available in this article, unless otherwise stated in a credit line to the data. 


\section{Background}

Knee osteoarthritis is a prevalent disease affecting up to $19 \%$ of the population over 45 years of age, causing chronic pain, disability, and lower quality of life [1-8].

Unicompartmental knee arthroplasty (UKA) is a costeffective treatment for femorotibial unicompartmental knee osteoarthritis. Over the last few years, the proportion of UKA had been increasing according to worldwide national registers [9-14]. Patients undergoing UKA had a lower rate of complications, faster functional recovery, and better satisfaction compared to total knee arthroplasty (TKA) [15-18]. Nevertheless, UKA has its difficulties. To achieve an adequate position of the components - crucial in avoiding early aseptic loosening - is more challenging than in TKA. Robotic assistance may play an essential role in decreasing the rate of malposition of the components in UKA [19, 20], theoretically leading to better clinical results and longer survival rates [21-27].

Few publications describe the radiological findings after robotic-assisted UKA using NAVIO (Blue Belt Technologies, Plymouth, MN, USA) [28]. This study aims to evaluate the precision of component position using five radiological parameters in conventional and robotic-assisted medial UKA using the NAVIO system. The hypothesis is that the robotic-assisted technique has a better rate of success compared to conventional UKA. Secondary aims were to compare short-term patientreported outcomes and pain between groups and to analyze whether an adequate component position related to better short-term results in UKA.

\section{Methods}

\section{Patient selection}

A cohort study was designed for patients who underwent medial UKA between April 2017 and March 2019 in a single center. The local Ethical Committee approved the study, and all patients signed, written informed consent before enrollment.

All patients' clinical records were reviewed, and their pre- and post-operative radiographs were measured. All patients undergoing medial UKA, having undergone a pre- and post-operative radiological study were included. Patients were excluded if they had an incomplete documentation or refused to participate.

Patients were allocated in the conventional (UKA-C) or robotic-assisted (UKA-R) group according to the surgeon's and patient's preference and the availability of the robot at the time of the surgery. In all patients, the Journey UNI implant (Smith \& Nephew Inc., Cordova, TN, USA) was used. All procedures were performed under spinal anesthesia by two senior knee surgeons (JD, R.N.). UKA-C was defined as the surgery performed without robotic assistance, and UKA-R as the surgery performed with the assistance of the NAVIO Robotic System (Blue
Belt Technologies, Plymouth, MN, USA). With the use of a tourniquet, an anterior approach and a medial parapatellar arthrotomy were performed in all surgeries. No wound drain was used.

The variables analyzed were age, gender, length of hospital stay, surgical time, and radiological measurements. The time of surgery in the UKA-C group ranges from incision to wound closure; meanwhile, in the UKA-R group, it was from the positioning of the pins (before the surgical incision) to wound closure.

\section{Radiological measurements}

Radiological measurements were performed using the immediate post-operative radiographs. An anteroposterior (AP) and a lateral knee radiograph were performed in all patients on day 1 after surgery. Two blinded orthopedic surgeons (NR, NJ) carried out the measurements.

The same protocol used in the study by Iñiguez et al. [19] was used (Fig. 1). The anatomic medial distal femoral angle (aMDFA) was defined as the angle between a line through the anatomical axis of the femur and a line that joins the most distal point of the lateral condyle and the medial femoral component. The sagittal femoral angle (SFA) was defined as the angle between a line through the anterior cortex of the metaphysealdiaphyseal junction and a line through the posterior peg of the femoral component.

The anatomical medial proximal tibial angle (aMPTA) was defined as the angle between a line through the anatomical axis of the tibia and a line through the tibial component. The tibial slope was defined as the angle between a line through the sagittal mechanical axis of the tibia and a line through the tibial component.

The femoral and tibial component size was assessed in the lateral knee radiograph, according to the designer classification: Oversized was defined when the implant protrudes more than $2 \mathrm{~mm}$ and undersized when the implant does not achieve adequate coverage. The target for each measurement was defined as follows: tibial slope $5 \pm 3^{\circ}$, aMDFA $98 \pm 3^{\circ}$, aMPTA $87 \pm 3^{\circ}$, SFA $45 \pm 3^{\circ}$ and an adequate component size.

\section{Clinical measurements}

All patients were evaluated pre-surgery, and then they were contacted 6 months after surgery by a blinded evaluator to request that they complete the Oxford Knee Score (OKS) and to evaluate knee pain at rest using a numeric rating scale (NRS) from 0 to 10 , with 0 being no pain.

\section{Statistics}

The frequency, proportions, median, range, and interquartile range were used to describe the sample of the study. The nonparametric median test was used to 


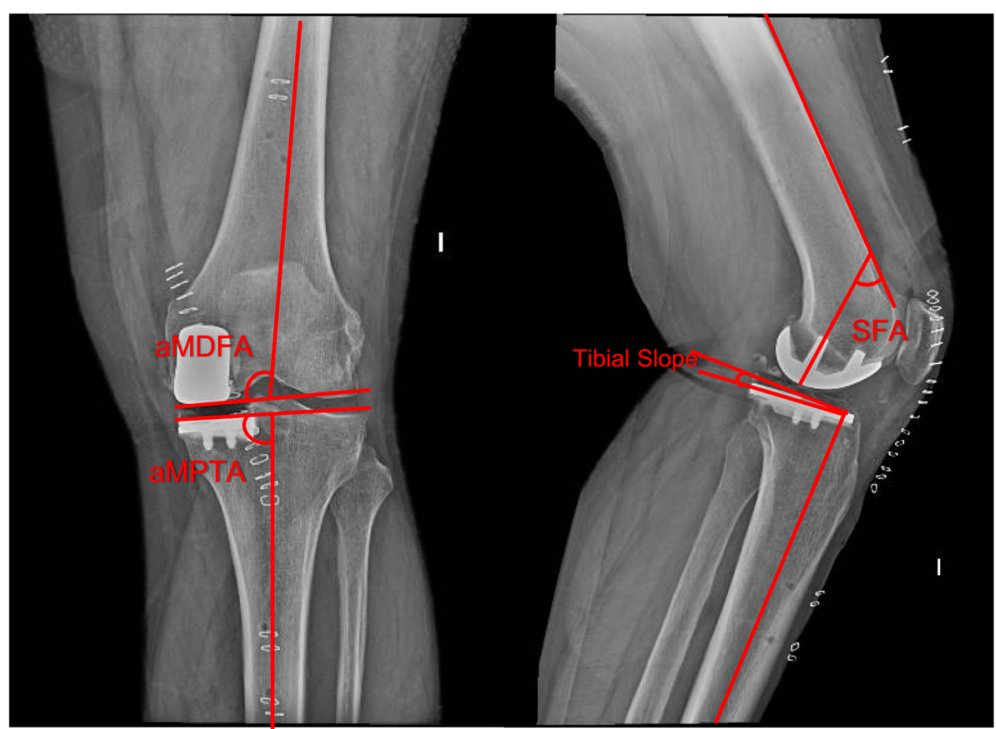

Fig. 1 Radiological measures in the anteroposterior and lateral knee x-ray. aMDFA anatomical medial distal femoral angle, aMPTA anatomical medial proxial tibial angle, SFA sagital femoral angle

compare the continuous variables: age, affected knee side, length of hospital stay, surgery time, OKS, NRS, and radiographic measurements. Fisher's exact test was used to compare categorical variables: gender, side, and component size. Multivariate logistic discrimination analysis was conducted to assess the capacity to recognize whether the UKA was robotic-assisted or conventional using the radiological parameters as predictor variables.

The frequency of success in each radiological measurement was compared between groups using a Fisher's exact test. A successful UKA was considered if the target was achieved in at least four of the radiographic parameters. The proportion of successful UKAs was compared between groups using Fisher's exact test. The power of the estimation $(1-\beta)$ was reported and considered underpowered if $1-\beta$ was lower than 0.8. Also, to analyze the relevance of a successful UKA, a nonparametric median test was used to compare the median OKS and pain NRS between successful and failed UKAs.

Confidence intervals of $\pm 95 \%$ were built, and a significance level of 0.05 was used. The data were processed using Stata 11.2 version (StataCorp LP, College Station, TX, USA).

\section{Results}

A total of 34 patients were included, of whom 18 were UKA-R patients (Table 1). No significant differences were found between the two groups in terms of gender, age, affected knee side, or pre-operative OKS or pain NRS (Tables 1 and 2). Also, no differences were found in pre-operative radiological measurements. The median total surgery time was significantly lower in UKA-C patients $(p<0.01)$ (Table 2).

The post-operative radiographic measurements are summarized in Table 2. Only the SFA reached a significant difference (nonparametric median test, $p=0.02$ ) between groups. The ability to discriminate between groups using the radiological parameters was high, being the error classification error 0.11. Only one UKA-R was misclassified as UKA-C, but two UKA-Cs were misclassified as UKA-R.

The rate of success to achieve the desired target in each radiological measurement is summarized in Table 3. Successful UKA was achieved in 14 (88\%) of the UKA-R patients, but only five (28\%) of the UKA-C patients (Table 4). The proportion of successful outcomes was statistically significant and powered (Fisher exact test, $p=0.001 ; 1-\beta=0.95)$.

A significantly better OKS was achieved in the UKA-R group (nonparametric median test, $p=0.01$ ) (Fig. 2). Yet, the median difference between groups was only 5 points, so it may not be clinically relevant (Table 2). The

Table 1 Comparison of demographics variables between groups

\begin{tabular}{lllll}
\hline & UKA-R & UKA-C & Total & $\boldsymbol{P}$ \\
\hline $\boldsymbol{N}$ & $16(47 \%)$ & $18(53 \%)$ & 34 & N/A \\
Male & $7(44 \%)$ & $12(67 \%)$ & $19(56 \%)$ & $0.16^{*}$ \\
Age & $66(56$ to 82$)$ & $65(41$ to 76$)$ & $66(41$ to 82$)$ & $0.60^{* *}$ \\
Right side & $10(63 \%)$ & $8(44 \%)$ & $16(47 \%)$ & $0.32^{*}$ \\
\hline
\end{tabular}

UKA- $R$ robotic-assisted unicompartmental knee arthroplasty UKA-C conventional unicompartmental knee arthroplasty, $N$ number *Fisher's exact test, **nonparametric test median 
Table 2 Comparison of clinical and radiological measurements between groups

\begin{tabular}{llll}
\hline & UKA-R & UKA-C & P \\
\hline BS OKS & $17(6-41)[11-23]$ & $18(12-30)[15-21]$ & $0.34^{* *}$ \\
BS Pain NRS & $6(2-10)[5-7]$ & $7(2-10)[6-8]$ & $0.52^{* *}$ \\
Lengh of hospital stay (days) & $2(1-4)[2-3]$ & $2(1-3)[2-2]$ & $0.25^{* *}$ \\
Surgery time & $139(125-156)[129-72]$ & $106(85-131)[98-124]$ & $<0.01^{* *}+$ \\
Sagital femoral angle & $46(42-57)[44-47]$ & $48(33-65)[44-53]$ & $0.47^{* *}$ \\
aMDFA & $98(95-101)[97-99]$ & $101(96-105)[98-103]$ & $0.02^{* *+}$ \\
aMPTA & $86.9(83-92)[85-88]$ & $85.3(81-97)[83-86]$ & $0.26^{* *}$ \\
Slope & $4.4(1.7-7.0)[3.3-5.1]$ & $5.3(0.7-10.0)[3.8-6.9]$ & $0.09^{* *}$ \\
Incorrect size & $0(0 \%)$ & $4(22 \%)$ & $0.07^{*}$ \\
Post-operative OKS & $45(37-47)[41-47]$ & $39(23-48)[37-42]$ & $0.01^{* *}+$ \\
Post-operative pain NRS & $1(0-3)[0-1]$ & $4(0-9)[2-6]$ & $<0.01^{* *}+$ \\
\hline
\end{tabular}

UKA-R robotic-assisted unicompartmental knee arthroplasty, UKA-C conventional unicompartmental knee arthroplasty, BS before surgery, NRS numerical rating scale, aMDFA anatomical medial distal femoral angle, aMPTA anatomical medial proximal tibial angle, OKS Oxford Knee Score

*Fisher's exact test; ${ }^{* *}$ nonparametric test median; + significant difference

median pain NRS in the UKA-R was 1 (range, 0 to 3 ) and 4 in the UKA-C (Fig. 3); this difference reached statistical significance (nonparametric median test, $p<$ 0.000).

Successful UKA had a trend towards a higher OKS (nonparametric median test, $p=0.088 ; 1-\beta=0.51$ ) and a significantly lower pain NRS (nonparametric median test, $p=0.036 ; 1-\beta=0.57$ ), but both findings were underpowered.

There was only one complication in the UKA-C group; this was an arthrofibrosis that required mobilization under anesthesia. No complications were found in the UKA-R group.

\section{Discussion}

The main finding of this study is that UKA-R yielded a significantly higher rate of radiological successful UKA. Also, there was a trend towards a better functional patient-reported outcome and less pain during rest. Moreover, the definition of successful UKA by the radiological measurements used in this study was significantly

Table 3 Frequency that each group achieved the desired target in radiological parameters

\begin{tabular}{llll}
\hline & UKA-R & UKA-C & $\boldsymbol{P}^{*}$ \\
\hline aMDFA 98 $\pm \mathbf{3}^{\circ}$ & $14(88 \%)$ & $10(56 \%)$ & 0.06 \\
aMPTA $\mathbf{8 7} \pm \mathbf{3}^{\circ}$ & $13(81 \%)$ & $11(61 \%)$ & 0.27 \\
Tibial slope $\mathbf{5} \pm \mathbf{3}^{\circ}$ & $15(94 \%)$ & $15(83 \%)$ & 0.60 \\
Sagittal femoral angle $\mathbf{4 5} \pm \mathbf{3}^{\circ}$ & $12(75 \%)$ & $5(28 \%)$ & $0.02+$ \\
Correct implant size & $16(100 \%)$ & $14(78 \%)$ & 0.11
\end{tabular}

UKA-R robotic-assisted unicompartmental knee arthroplasty, UKA-C conventional unicompartmental knee arthroplasty, aMDFA anatomical medial distal femoral angle, aMPTA anatomical medial proximal tibial angle *Fisher's exact test, + significant difference related to better functional outcomes and lower pain levels during rest, yet this finding was underpowered. This study is a continuation of the clinical phase in the cadaveric pilot study published by our team, which showed consistent results of greater accuracy in relation to implant position in robotic-assisted surgery, compared to the conventional technique [19].

Published studies have shown that robotic-assisted surgery allows for greater accuracy and improved implant position [29-33].

In the radiological assessment of the cases studied, we considered five imaging parameters and we defined success as the fulfillment of at least four out of five of the requirements since we believe that combining all the radiological parameters achieved a more comprehensive way of measuring accuracy. We have no references to previous publications in which this way of assessing

Table 4 The number of desired targets achieved by each patient who underwent UKA. Successful UKA was defined when at least four radiological parameters reached the desired goal. UKA-C achieved a success rate of 0.28 ; meanwhile, UKA-R achieved a rate of 0.88 . The proportion of successful outcomes was statistically significant and powered (Fisher's exact test, $p=$ $0.001 ; 1-\beta=0.95)$

\begin{tabular}{lll}
\hline $\boldsymbol{N}^{\circ}$ of target achieved & UKA-R & UKA-C \\
\hline $\mathbf{5}$ & $9(56 \%)$ & $3(17 \%)$ \\
$\mathbf{4}$ & $5(31 \%)$ & $2(11 \%)$ \\
$\mathbf{3}$ & $2(13 \%)$ & $8(44 \%)$ \\
$\mathbf{2}$ & 0 & $4(22 \%)$ \\
$\mathbf{1}$ & 0 & $1(06 \%)$ \\
$\mathbf{0}$ & 0 & 0 \\
\hline
\end{tabular}

UKA- $R$ robotic-assisted unicompartmental knee arthroplasty, UKA-C conventional unicompartmental knee arthroplasty, $N$ number $\boldsymbol{N}^{\circ}$ Number of targets achieved 
UKA-C

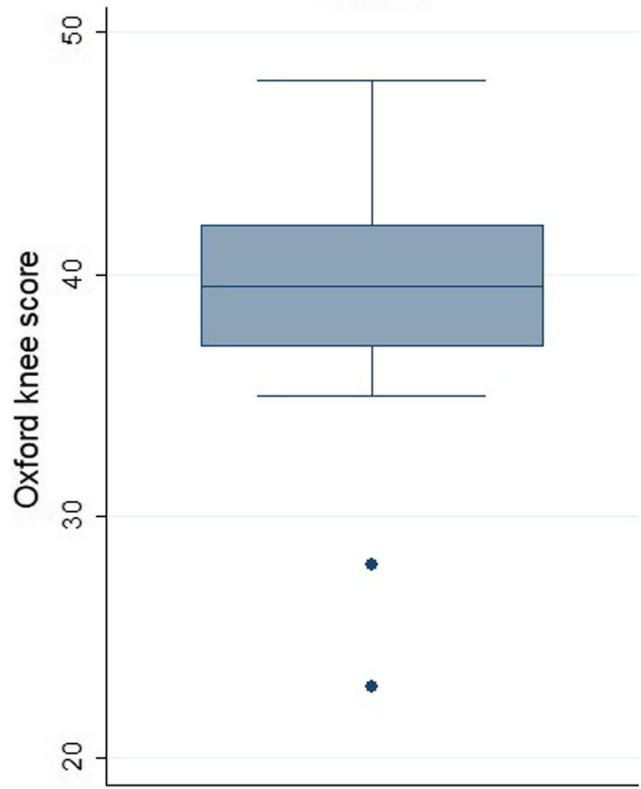

UKA-R

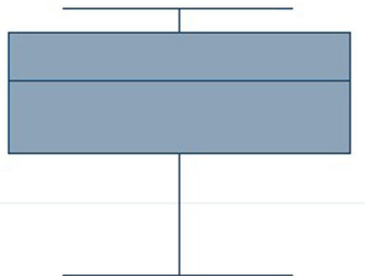

Fig. 2 Distribution of Oxford Knee Score (OKS) among groups. Robotic-assisted unicompartmental knee arthroplasty (UKA-R) had a trend to better OKS than conventional unicompartmental knee arthroplasty (UKA-C) (nonparametric median test, $p=0.01$ )

accuracy is used, and considering the prosthesis as a whole and not analyzing each radiological variable in particular seem to provide a more comprehensive evaluation.

By analyzing the results of the radiological measurements in both groups separately, there are only statistically significant differences among them in terms of SFA. However, when considering the objective of success with the predefined parameters, the robotic group reaches $88 \%$ versus $28 \%$ for the conventional group ( $p=$ 0.001 ). This correlates with the logistic discriminant analysis of both groups, which shows an error rate of 0.11,

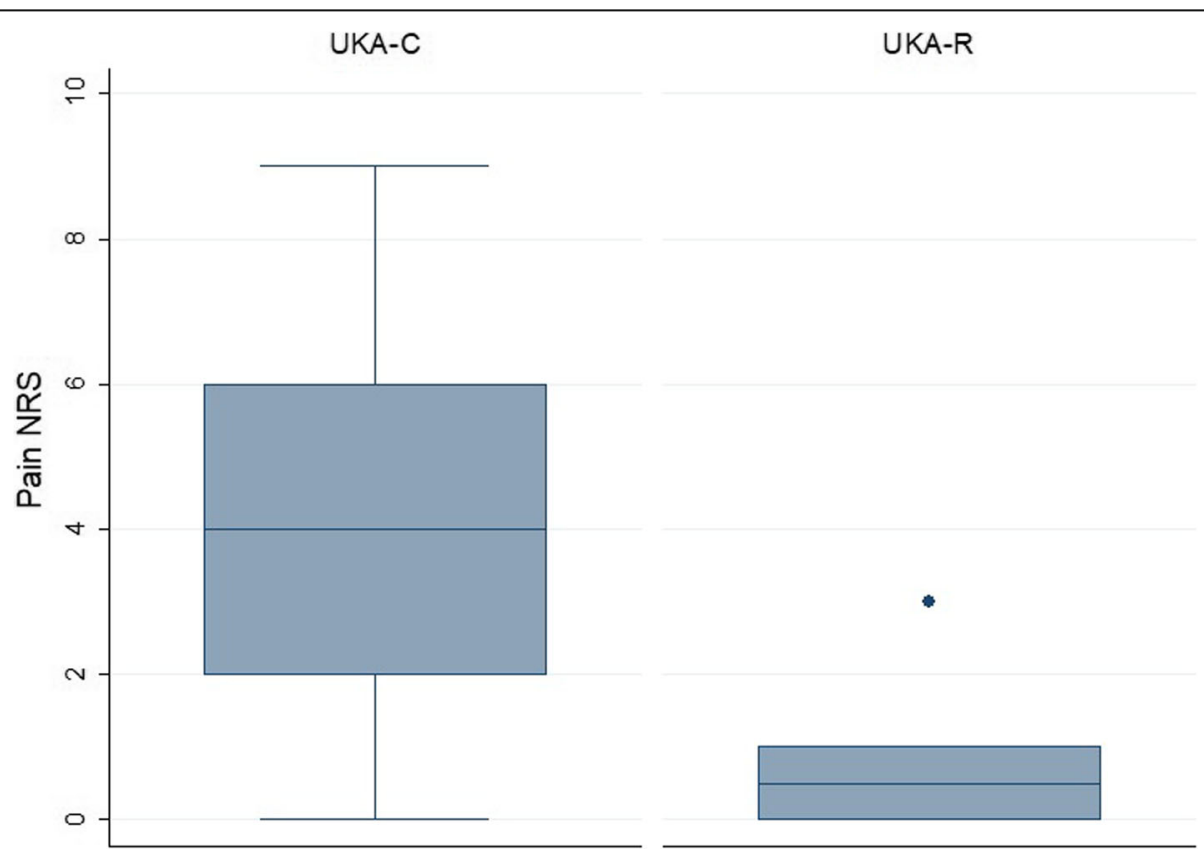

Fig. 3 Distribution of numerical rating scale (NRS) for pain among groups. Robotic-assisted unicompartmental knee arthroplasty (UKA-R) had a trend to lower NRS for pain than conventional unicompartmental knee arthroplasty (UKA-C) (nonparametric median test, $p<0.000$ ) 
with only one UKA-R wrongly categorized as conventional. We defined an interval of $\pm 3^{\circ}$ as it was the interval that maximized the difference between the groups. Bell et al. published a difference with an interval of $\pm 2^{\circ}$ [34]; however, in our study using an interval of $\pm 1^{\circ}$ more in width we found a significant difference.

The first published data on the accuracy of implant positioning using the NAVIO system were promising. Batailler et al. published the first clinical study comparing robotic-assisted UKA versus the conventional technique [24]. The authors conclude that there is a significant improvement in the accuracy of implant positioning with robotic-assisted surgery in the coronal as well as in the sagittal plane, thus reducing the number of outliers, but no significant difference was found in functional results among the groups studied. Our results were similar to those published by Batallier et al. regarding the accuracy of implant positioning, although we did find better functional and post-operative pain results in the UKA-R group compared to the UKA-C group at 1year follow-up [24], which was similar to results published in other series [20] of robotic-assisted surgery.

In this series, we did not find any complications in either group, with just one case of manipulation under anesthesia in the conventional group that could explain the difference in the OKS and NRS in favor of the robotic group. This is different from what St. Mart reported; in his study he had a higher rate of revision in the robotic group because of early infection [35]. The fact that we did not find any complications in the robotic group makes us believe that this is a safe procedure, even though these were our first cases, which was similar to what Mergenthaler [36] published in his casecontrol series.

The limitations of this study include its small sample size and short-term follow-up of 6 months, and not the 2-year follow-up that most studies report, which prevented us from adequately demonstrating some of the trends observed and does not necessarily represent longterm differences. There also may be a bias for the pain NRS score, and this may explain why the results of the NRS in conventional surgery are higher than those reported in the literature. Another limitation is the way that accuracy is measured with $\mathrm{x}$-rays, which does not allow us to assess the rotational effects on implants. In the study design, we rejected the use of computed tomgraphy scans due to the need for patient exposure to radiation which is equivalent to 48 chest $\mathrm{x}$-rays, as Ponzio et al. [37] pointed out, and also due to the high cost associated with it, cost being one of the advantages of the imageless robotic NAVIO system used.

Future studies in larger population groups and longterm follow-ups are necessary to confirm the trend observed in the favorable results of our study.

\section{Conclusions}

Robotic-assisted UKA with the NAVIO system offers greater accuracy of femoral implant positioning in the sagittal plane, and it is more accurate in achieving clinical and radiological success compared to conventional surgery.

\section{Acknowledgements \\ To the Department of Orthopaedics and Traumatology for its support.}

\section{Authors' contributions}

RN: conceptualization, methodology, resources, supervision, project administration. JD: conceptualization, resources, supervision, project administration. MI: methodology, data curation, writing - review and editing. NOR: methodology, validation, writing - review and editing, visualization. MB: methodology, formal analysis. Cl: methodology, resources, writing review and editing. GF: conceptualization, supervision. NJ: investigation, writing - original draft, visualization. The author(s) read and approved the final manuscript.

Funding

Not applicable

\section{Availability of data and materials}

All data generated or analyzed during this study are included in this published article and its supplementary information files.

\section{Ethics approval and consent to participate}

The local Ethical Committee approved the study, and all patients signed written, informed consent before enrollment.

Consent for publication

Not applicable

\section{Competing interests}

The authors declare that they have no competing interests related to this study.

\section{Author details}

'Department of Orthopedics and Traumatology, Clinica Las Condes, Estoril 450, Las Condes, Santiago, Chile. ${ }^{2}$ Department of Orthopaedic Surgery, Hospital Clinico de la Universidad de Chile, Santiago, Chile.

Received: 3 August 2020 Accepted: 12 January 2021

Published online: 12 February 2021

References

1. Lawrence RC, Felson DT, Helmick CG, Arnold LM, Choi H, Deyo RA, Gabriel S, Hirsch R, Hochberg MC, Hunder GG, Jordan JM, Katz JN, Kremers HM, Wolfe F, National Arthritis Data Workgroup (2008) Estimates of the prevalence of arthritis and other rheumatic conditions in the United States: part II. Arthritis Rheum 58:26-35

2. Vos T, Flaxman AD, Naghavi M et al (2012) Years lived with disability (YLDs) for 1160 sequelae of 289 diseases and injuries 1990-2010: a systematic analysis for the Global Burden of Disease Study 2010. Lancet 380:2163-2196

3. Bijlsma JWJ, Berenbaum F, Lafeber FPJG (2011) Osteoarthritis: an update with relevance for clinical practice. Lancet 377:2115-2126

4. Nguyen US, Zhang Y, Zhu Y, Niu J, Zhang B, Felson DT (2011) Increasing prevalence of knee pain and symptomatic knee osteoarthritis: survey and cohort data. Ann Intern Med 155(11):725-732

5. Felson DT, Lawrence RC, Dieppe PA, Hirsch R, Helmick CG, Jordan JM, Kington RS, Lane NE, Nevitt MC, Zhang Y, Sowers M, McAlindon T, Spector TD, Poole AR, Yanovski SZ, Ateshian G, Sharma L, Buckwalter JA, Brandt KD, Fries JF (2000) Osteoarthritis: new insights. Part 1: the disease and its risk factors. Ann Intern Med 133(8):635-646

6. Loeser RF, Collins JA, Diekman BO (2016) Ageing and the pathogenesis of osteoarthritis. Nat Rev Rheumatol 12:412-420

7. Abbott JH, Usiskin IM, Wilson R, Hansen P, Losina E (2017) The quality-of-life burden of knee osteoarthritis in New Zealand adults: a model-based evaluation. PLoS One 12(10):e0185676 
8. Wallace IJ, Worthington S, Felson DT, Jurmain RD, Wren KT, Maijanen $H$, Woods RJ, Lieberman DE (2017) Knee osteoarthritis has doubled in prevalence since the mid-20th century. Proc Natl Acad Sci U S A 114(35): 9332-9336

9. Bolognesi MP, Greiner MA, Attarian DE, Watters TS, Wellman SS, Curtis LH, Berend KR, Setoguchi S (2013) Unicompartmental knee arthroplasty and total knee arthroplasty among Medicare beneficiaries, 2000 to 2009. J Bone Joint Surg Am 95(22):e174

10. Swank ML, Alkire M, Conditt M, Lonner JH (2009) Technology and costeffectiveness in knee arthroplasty: computer navigation and robotics. Am J Orthop (Belle Mead NJ) 38(2 Suppl):32-36

11. Australian Orthopaedic Association National Joint Registry. Hip and knee arthroplasty annual report 2018. https://aoanjrr.sahmri.com/annualreports-2018

12. New Zealand Joint Registry. The New Zealand Registry annual report. 2018. https://nzoa.org.nz/system/files/DH8152_NZJR_2018_Report_v6_4Decv18.pdf

13. Swedish Knee Arthroplasty Register. Annual report - Swedish Knee Arthroplasty Register 2018. https://www.researchgate.net/publication/329566953_The_ Swedish_Knee_Arthroplasty_Register_-_Annual_Report_2018

14. National Joint Registry for England, Wales and Northern Ireland. 15th annual report 2018. https://www.hqip.org.uk/wp-content/uploads/2018/11/NJR-15 th-Annual-Report-2018.pdf. Accessed 15 July 2019.

15. Schwab PE, Lavand'homme P, Yombi JC, Thienpont E (2015) Lower blood loss after unicompartmental than total knee arthroplasty. Knee Surg Sports Traumatol Arthrosc 23(12):3494-3500

16. Watanabe T, Abbasi AZ, Conditt MA, Christopher J, Kreuzer S, Otto JK, Banks SA (2014) In vivo kinematics of a robot-assisted uni- and multicompartmental knee arthroplasty. J Orthop Sci 19(4):552-557

17. Larsen K, Sørensen OG, Hansen TB, Thomsen PB, Søballe K (2008) Accelerated perioperative care and rehabilitation intervention for hip and knee replacement is effective: a randomized clinical trial involving 87 patients with 3 months of follow-up. Acta Orthop 79(2):149-159

18. McAllister CM (2008) The role of unicompartmental knee arthroplasty versus total knee arthroplasty in providing maximal performance and satisfaction. J Knee Surg 21(4):286-292

19. Iñiguez M, Negrín R, Duboy J, Reyes NO, Díaz R (2019) Robot-assisted unicompartmental knee arthroplasty: increasing surgical accuracy? A cadaveric study. J Knee Surg. https://doi.org/10.1055/s-0039-1698771 [published online ahead of print, 2019 Oct 22]

20. van der List JP, Chawla H, Joskowicz L, Pearle AD (2016) Current state of computer navigation and robotics in unicompartmental and total knee arthroplasty: a systematic review with meta-analysis. Knee Surg Sports Traumatol Arthrosc 24(11):3482-3495

21. Barbadoro P, Ensini A, Leardini A, d'Amato M, Feliciangeli A, Timoncini A, Amadei F, Belvedere C (2014) Giannini S Tibial component alignment and risk of loosening in unicompartmental knee arthroplasty: a radiographic and radiostereometric study. Knee Surg Sports Traumatol Arthrosc 22(12):3157-3162

22. Jenny JY, Boeri C (2003) Unicompartmental knee prosthesis implantation with a non-image-based navigation system: rationale, technique, casecontrol comparative study with a conventional instrumented implantation. Knee Surg Sports Traumatol Arthrosc 11(1):40-45

23. Pearle AD, van der List JP, Lee L, Coon TM, Borus TA, Roche MW (2017) Survivorship and patient satisfaction of robotic-assisted medial unicompartmental knee arthroplasty at a minimum two-year follow-up. Knee 24(2):419-428

24. Batailler C, White N, Ranaldi FM, Neyret P, Servien E, Lustig S (2019) Improved implant position and lower revision rate with robotic-assisted unicompartmental knee arthroplasty. Knee Surg Sports Traumatol Arthrosc 27(4):1232-1240

25. Kleeblad LJ, Borus TA, Coon TM, Dounchis J, Nguyen JT, Pearle AD (2018) Midterm survivorship and patient satisfaction of robotic-arm-assisted medial unicompartmental knee arthroplasty: a multicenter study. J Arthroplasty 33(6):1719-1726

26. Winnock de Grave P, Barbier J, Luyckx T, Ryckaert A, Gunst P, Van den Daelen $L$ (2018) Outcomes of a fixed-bearing, medial, cemented unicondylar knee arthroplasty design: survival analysis and functional score of 460 cases. J Arthroplasty 33(9):2792-2799

27. Lombardi AV Jr, Berend KR, Walter CA, Aziz-Jacobo J, Cheney NA (2009) Is recovery faster for mobile-bearing unicompartmental than total knee arthroplasty? Clin Orthop Relat Res 467(6):1450-1457
28. Fu J, Wang Y, Li X et al (2018) Robot-assisted vs. conventional unicompartmental knee arthroplasty: systematic review and meta-analysis Roboterassistierte vs. konventionelle unikompartimentäre knieendoprothese: systematisches review und metaanalyse. Orthopade 47(12):1009-1017

29. Robinson PG, Clement ND, Hamilton D, Blyth MJG, Haddad FS, Patton JT (2019) A systematic review of robotic-assisted unicompartmental knee arthroplasty: prosthesis design and type should be reported. Bone Joint J 101-B(7):838-847

30. Cobb J, Henckel J, Gomes P, Harris S, Jakopec M, Rodriguez F, Barrett A, Davies B (2006) Hands-on robotic unicompartmental knee replacement: a prospective, randomised controlled study of the acrobot system. J Bone Joint Surg Br 88(2):188-197

31. Weber P, Crispin A, Schmidutz F, Utzschneider S, Pietschmann MF, Jansson V, Müller PE (2013) Improved accuracy in computer-assisted unicondylar knee arthroplasty: a meta-analysis. Knee Surg Sports Traumatol Arthrosc 21(11):2453-2461

32. Lonner JH, Smith JR, Picard F, Hamlin B, Rowe PJ, Riches PE (2015) High degree of accuracy of a novel image-free handheld robot for unicondylar knee arthroplasty in a cadaveric study. Clin Orthop Relat Res 473(1):206-212

33. Blyth MJG, Anthony I, Rowe P, Banger MS, MacLean A, Jones B (2017) Robotic arm-assisted versus conventional unicompartmental knee arthroplasty: exploratory secondary analysis of a randomised controlled trial. Bone Joint Res 6(11):631-639

34. Bell SW, Anthony I, Jones B, MacLean A, Rowe P, Blyth M (2016) Improved accuracy of component positioning with robotic-assisted unicompartmental knee arthroplasty: data from a prospective, randomized controlled study Bone Joint Surg Am 98(8):627-635. https://doi.org/10.2106/JBJS.15.00664

35. St Mart JP, de Steiger RN, Cuthbert A, Donnelly W (2020) The three-year survivorship of robotically assisted versus non-robotically assisted unicompartmental knee arthroplasty. Bone Joint J 102-B(3):319-328

36. Mergenthaler G, Batailler C, Lording T, Servien E, Lustig S (2020) Is roboticassisted unicompartmental knee arthroplasty a safe procedure? A case control study. Knee Surg Sports Traumatol Arthrosc. https://doi.org/10.1007/ s00167-020-06051-Z

37. Ponzio DY, Lonner JH (2015) Preoperative mapping in unicompartmental knee arthroplasty using computed tomography scans is associated with radiation exposure and carries high cost. J Arthroplasty 30(6):964-967

\section{Publisher's Note}

Springer Nature remains neutral with regard to jurisdictional claims in published maps and institutional affiliations.

Ready to submit your research? Choose BMC and benefit from:

- fast, convenient online submission

- thorough peer review by experienced researchers in your field

- rapid publication on acceptance

- support for research data, including large and complex data types

- gold Open Access which fosters wider collaboration and increased citations

- maximum visibility for your research: over $100 \mathrm{M}$ website views per year

At $\mathrm{BMC}$, research is always in progress.

Learn more biomedcentral.com/submissions 